\title{
SOME RESULTS CONCERNING THE CAUCHY FUNCTIONAL EQUATION IN CERTAIN BANACH ALGEBRAS
}

\author{
J. VUKMAN
}

\begin{abstract}
In this paper some results concerning the Cauchy functional equation, that is the functional equation $f(x+y)=f(x)+f(y)$ in complex hermitian Banach *-algebras with an identity element are presented. As an application a generalization of Kurepa's extension of the Jordan-Neumann characterization of pre-Hilbert space is obtained.
\end{abstract}

\section{Preliminaries}

All algebras and vector spaces in this paper will be over the complex field. Algebras are assumed to have an identity element, which will be denoted by $e$. An algebra $A$ is called a *algebra if there exists an involution (conjugate-linear anti-isomorphism of period two) $a \mapsto a^{*}$ on $A$. An element $h \in A$ is said to be hermitian if $h^{*}=h$, and $a \in A$ will be called normal if $a^{*} a=a a^{*}$. It is easy to see that each element $a \in A$ has a unique decomposition $a=h+i k$ with hermitian $h$ and $k$.

A *-algebra which is also a Banach algebra is called a Banach *algebra. A Banach *-algebra is called hermitian if each hermitian element has real spectrum. Let $A$ be a hermitian Banach *-algebra and let $h \in A$ be a hermitian element. It is convenient to write $h>0$ if

Received 18 September 1984 .

Copyright Clearance Centre, Inc. Serial-fee code: 0004-9727/85 $\$ A 2.00+0.00$. 
the spectrum of $h$ is positive. The most important hermitian Banach *-algebras are $B^{*}$-algebras (that is Banach *algebras in which $\left\|a^{*} a\right\|=\|a\|^{2}$ is fulfilled for all $a$ ). For the basic facts concerning hermitian Banach *algebras, we refer the reader to Ptak's paper [5].

Let $X$ and $A$ be a vector space and an algebra, respectively. Suppose that $X$ is a left A-module. A left A-module $X$ will be called unitary if $e x=x$ holds for all $x \in X$.

\section{The Cauchy functional equation}

The Cauchy functional equation, that is the functional equation $f(x+y)=f(x)+f(y)$ has been extensively studied (see [3], [6] and also [1] where further references can be found). In this section we present some results concerning the Cauchy functional equation on hermitian Banach *-algebras. We shall need the following.

LEMMA 1.1. Let $A$ be a hermitian Banach *algebra. For each $h>0$ there corresponds $u>0$, such that $u^{2}=h$. Moreover, $u$ commutes with each element which commutes with $h$.

Proof. A simple consequence of Ford's square root lemma [2] (see also $[5,(1.5)])$.

First we shall prove the following result.

LEMMA 1.2. Let A be a hermitian Banach *-algebra. Suppose there exists an additive fronction $f: A \rightarrow A$ such that $f(e)=0$ and that $f(a)=a f\left(a^{-1}\right) a^{*}$ holds for all invertible normal elements $a \in A$. In this case $f(a)=0$ for all $a \in A$.

Proof. Let us first prove that

$$
f\left(h^{2}\right)=0
$$

for all invertible hermitian elements $h \in A$. We have 


$$
\begin{aligned}
f\left(h^{2}\right) & =f\left(e+h^{2}\right)=\left(e+h^{2}\right) f\left(\left(e+h^{2}\right)^{-1}\right)\left(e+h^{2}\right) \\
& =\left(e+h^{2}\right) f\left(e-\left(e+h^{2}\right)^{-1} h^{2}\right)\left(e+h^{2}\right) \\
& =-\left(e+h^{2}\right) f\left(\left(e+h^{2}\right)^{-1} h^{2}\right)\left(e+h^{2}\right) \\
& =-\left(e+h^{2}\right)\left(e+h^{2}\right)^{-1} h^{2} f\left(h^{-2}\left(e+h^{2}\right)\right) h^{2}\left(e+h^{2}\right)^{-1}\left(e+h^{2}\right) \\
& =-h^{2} f\left(e+h^{-2}\right) h^{2}=-h^{2} f\left(h^{-2}\right) h^{2}=-f\left(h^{2}\right) .
\end{aligned}
$$

Hence $f\left(h^{2}\right)=-f\left(h^{2}\right)$ which proves (1). From (1) it follows that

$$
f(t e)=0
$$

holds for all real numbers $t$. Let us prove that

$$
f(h)=0
$$

is fulfilled for all hermitian $h \in A$. Let therefore $h \in A$ be an arbitrary hermitian element. Let us choose a real number $t$ such that $t e+h>0$. By Lemma 1.1 there exists an invertible hermitian element

$u \in A$ such that $t e+h=u^{2}$. According to (1) and (2) we have $f(h)=f(t e+h)=f\left(u^{2}\right)=0$ which proves $(3)$. Now we intend to prove that

$$
f(i h)=0
$$

for all invertible hermitian elements $h \in A$. We have

$$
\begin{aligned}
f(i h) & =f(e+i h)=(e+i h) f\left((e+i h)^{-1}\right)(e-i h) \\
& =i e+i h ; f\left(e-(e+i h)^{-1} i h\right)(e-i h) \\
& =-(e+i h) f\left((e+i h)^{-i} i h\right)(e-i h) \\
& =-(e+i h)(e+i h)^{-1} i h f\left((i h)^{-1}(e+i h)\right)(-i h)(e-i h)^{-1}(e-i h) \\
& \left.=-i h f(e+i, i h)^{-1}\right)(-i h)=-i h f\left((i h)^{-1}\right)(-i h)=-f(i h) .
\end{aligned}
$$

Hence $f(i h)=-f(i h)$ which proves the relation $(4)$.

From (4) it follows that

$$
f(i t e)=0
$$

holds for all real numbers $t$. Let us prove that

$$
f(i h)=0
$$

is fulfilled for all hermitian $h \in A$. Let therefore $h \in A$ be an arbitrary hermitian element and let $t$ be such a real number that 
$(t e+h)^{-1}$ exists. Then according to (4) and (5) it follows $f(i h)=f(i(t e+h))=0$ which proves the relation (6). Using (3) and (6) we obtain $f(a)=f(h+i k)=f(h)+f(i k)=0$ for all $a \in A$ which completes the proof of the theorem.

We shall need the result below in the next section.

THEOREM 1.3. Let A be a hermitian Banach *-algebra. Suppose there exists an additive function $f: A \rightarrow A$ such that $f(a)=a f\left(a^{-1}\right) a^{*}$ holds for all invertible normal elements $a \in A$. In this case $2 f(a)=a f(e)+f(e) a^{*}$ is fulfilled for all $a \in A$.

Proof. Obviously, the function $g: A \rightarrow A$ defined by the relation $g(a)=f(a)-\frac{1}{2}\left(a f(e)+f(e) a^{*}\right)$ is additive. A simple calculation shows that for all invertible normal elements $a \in A$ the relation $g(a)=a g\left(a^{-1}\right) a^{*}$ holds. Therefore, since $g(e)=0$, it follows from Lemma 1.2 that $g(a)=0$ is fulfilled for all $a \in A$ which completes the proof of the theorem.

We conclude this section with the theorem below.

THEOREM 1.4. Let $A$ be a herritian Banach *-algebra and $f: A \rightarrow A$ an additive fronction. The following statements are fulfilled:

(1) if $f(a)=a f\left(a^{-1}\right) a$ holds for all invertible normal elements $a \in A$ then $f$ is of the form $2 f(a)=a f(e)+f(e) a$;

(2) if $f(a)=a^{2} f\left(a^{-1}\right)$ holds for all invertible normal elements $a \in A$ then $f$ is of the form $f(a)=a f(e)$;

(3) if $f(a)=a^{*} a f\left(a^{-1}\right)$ holds for all invertible normal elements $a \in A$ then $f$ is of the form $f(a)=h f(e)$, where $a=h+i k$.

The proof of the results above is similar to the proof of Theorem 1.3 and will therefore be omitted. Let us point out that a result concerning the Cauchy functional equation in hermitian Banach *-algebras can be found in our earlier paper [7]. 


\section{A-bilinear and A-quadratic forms}

Let $X$ and $A$ be a vector space and a ${ }^{*}$-algebra, respectively. Suppose that $X$ is a left A-module. A mapping $B(\cdot, \cdot): X \times X \rightarrow A$ is called an A-bilinear form if

(1) $B\left(a_{1} x_{1}+a_{2} x_{2}, y\right)=a_{1} B\left(x_{1}, y\right)+a_{2} B\left(x_{2}, y\right), x_{1}, x_{2}, y \in X$, $a_{1}, a_{2} \in A$

(2) $B\left(x, a_{1} y_{1}+a_{2} y_{2}\right)=B\left(x, y_{1}\right) a_{1}^{*}+B\left(x, y_{2}\right) a_{2}^{*}, x, y_{1}, y_{2} \in X$, $a_{1}, a_{2} \in A$.

A mapping $Q: X \rightarrow A$ is called an A-quadratic form if

(3) $Q(x+y)+Q(x-y)=2 Q(x)+2 Q(y), x, y \in X$,

(4) $Q(a x)=a Q(x) a^{*}, x \in X, a \in A$.

Some examples of A-bilinear forms can be found in [7]. It is easy to see that each A-bilinear form gives rise to an A-quadratic form by the relation $Q(x)=B(x, x)$. We consider the following:

PROBLEM. Let $X$ and $A$ be a vector space and a *algebra, respectively. Suppose that $X$ is a left $A$-module, and that there exists an A-quadratic form $Q: X \rightarrow A$. Does there exist an A-bilinear form $B(\cdot, \cdot): X \times X \rightarrow A$ such that $Q(x)=B(x, x)$ holds for all $x \in X$ ?

Kurepa [4] has proved that the answer to the above question is affirmative if $A$ is the complex number field. His result can be considered as an extension of the well known result due to Jordan and von Neumann which characterizes pre-Hilbert spaces among all normed spaces. Vrbová [6] has obtained a simple proof of Kurepa's theorem. Using Theorem 1.3 and some ideas from [6] we prove the result below which generalizes Kurepa's result.

THEOREM 2.1. Let $X$ be a vector space and $A$ a hermitian Banach *algebra. Let $X$ be a unitary left A-module, and suppose that there exists an A-quadratic form $Q: X \rightarrow A$. In this case the mapping $B(\cdot, \cdot): X \times X \rightarrow A$ defined by

$$
B(x, y)=\frac{1}{4}(Q(x+y)-Q(x-y))+\frac{i}{4}(Q(x+i y)-Q(x-i y))
$$

is an A-bilinear form. For all $x \in X$ the relation $Q(x)=B(x, x)$ 
holds.

Proof. Similarly, as in the proof of Kurepa's result (see [4], [6] and also [7]) one can prove that the function $S(\cdot, \cdot)$ defined by the relation $S(x, y)=Q(x+y)-Q(x-y)$ is additive in both variables. From the fact that $S(\cdot, \cdot)$ is additive in both variables it follows that the same is true for the function $B(\cdot, \cdot)$ defined by the relation $B(x, y)=\frac{1}{2} S(x, y)+(i / 4) S(x, i y)$. It is easy to see that $Q(x)=B(x, x)$ holds for all $x \in X$. It remains to prove that

$$
B(a x, y)=a B(x, y), B(x, a y)=B(x, y) a^{*},
$$

is fulfilled for all pairs $x, y \in X$ and all $a \in A$. Now we shall use the condition

$$
z(a x)=a Q(x) a^{*} \text {. }
$$

First of all it follows from this condition that

$$
S(a x, y)=a S\left(x, a^{-1} y\right) a^{*}
$$

holds for all pairs $x, y \in X$ and all invertible elements $a \in A$. Indeed

$$
\begin{aligned}
a S\left(x, a^{-1} y\right) a^{*} & =a Q\left(x+a^{-1} y\right) a^{*}-a Q\left(x-a^{-1} y\right) a^{*} \\
& =Q(a x+y)-Q(a x-y) \\
& =S(a x, y) .
\end{aligned}
$$

Let us prove that $B(\cdot, \cdot)$ satisfies the following relations:

$$
\begin{aligned}
& B(i x, y)=i B(x, y), x, y \in X, \\
& B(x, i y)=-i B(x, y), x, y \in X .
\end{aligned}
$$

We have

$$
\begin{aligned}
4 B(i x, y) & =S(i x, y)+i S(i x, i y) \\
& =S(x,-i y)+i S(x, y) \\
& =i(S(x, y)-i S(x,-i y)) \\
& =i(S(x, y)+i S(x, i y)) \\
& =4 i B(x, y)
\end{aligned}
$$

which proves (10). Furthermore, 


$$
\begin{aligned}
4 B(x, i y) & =S(x, i y)+i S(x,-y) \\
& =S(x, i y)-i S(x, y) \\
& =-i(S(x, y)+i S(x, i y)) \\
& =-4 i B(x, y)
\end{aligned}
$$

which proves (11).

We shall prove that for the function $f: A \rightarrow A$, defined by the relation

$$
f(a)=B(a x, y)+B(x, a y)
$$

where $x$ and $y$ are fixed vectors, the requirements of Theorem 1.3 are fulfilled. The additivity of the function above follows from the fact that $B(\cdot, \cdot)$ is additive in both variables. It remains to show that $f(a)=a f\left(a^{-1}\right) a^{*}$ holds for all invertible $a \in A$. We have $4 a f\left(a^{-1}\right) a^{*}=a\left(S\left(a^{-1} x, y\right)+i S\left(a^{-1} x, i y\right)\right) a^{*}+a\left(S\left(x, a^{-1} y\right)+i S\left(x, a^{-1} i y\right)\right) a^{*}$.

Using the fact that $S(x, y)=S(y, x)$ holds for all pairs $x, y \in X$ (this follows from the relation $Q(-x)=Q(x)$ ) and according to (9) we obtain

$$
\begin{aligned}
4 a f\left(a^{-1}\right) a^{*} & =S(x, a y)+i s(x, a i y)+S(a x, y)+i s(a x, i y) \\
& =4 B(x, a y)+4 B(a x, y) \\
& =4 f(a) .
\end{aligned}
$$

Hence $f(a)=a f\left(a^{-1}\right) a^{*}$. Therefore since all requirements of Theorem 1.3 are fulfilled it follows that

$$
B(a x, y)+B(x, a y)=a B(x, y)+B(x, y) a^{*}
$$

holds for all pairs $x, y \in X$ and all $a \in A$. Substituting $i a$ for $a$ and using (10) and (11) we obtain that for all pairs $x, y \in X$ and all $a \in A$ the relation

$$
B(a x, y)-B(x, a y)=a B(x, y)-B(x, y) a^{*}
$$

holds. From (13) and (14) it follows that (7) holds and the proof of the theorem is complete.

Let us point out that the result above can be proved using the same - approach and weaker assumption that the relation $Q(a x)=a Q(x) a^{*}$ is fulfilled only for normal invertible elements. It should be mentioned that 
in the case that $A$ is commutative Theorem 2.1 reduces to a result proved in our earlier paper [7] using a somewhat more complicated approach.

\section{References}

[1] J. Aczél, Lectures on functional equations and their applications (Academic Press, New York and London, 1966).

[2] J.W.M. Ford, "A square root lemma for Banach *-algebras", J. London Math. Soc. 42 (1967), 521-522.

[3] S. Kurepa, "The Cauchy functional equation and scalar product in vector spaces", Glasnik Mat. Fiz.-Astr. 19 (1964), 23-36.

[4] S. Kurepa, "Quadratic and sesquilinear functionals", Glasnik Mat. Fiz.-Astr. 20 (1965), 79-92.

[5] V. Pták, "Banach algebras with involution", Manuscripta Math. 6 $(1972), 245-290$.

[6] P. Vrbová, "Quadratic and bilinear forms", Časopis Pést. Mat. 98 (1973), 159-161.

[7] J. Vukman, "A result concerning additive functions in hermitian Banach *-algebras and an application", Proc. Amer. Math. Soc. 91 (1984), $367-372$.

University of Maribor,

VEKS,

Razlagova 14,

62000 Maribor,

Yugoslavia. 